OPEN ACCESS

Edited by:

Lbachir Benmohamed, University of California, Irvine,

United States

Reviewed by:

Jennifer Ann Juno,

The University of Melbourne, Australia

Paul Goepfert,

University of Alabama at Birmingham, United States

*Correspondence:

Nimesh Gupta

nimesh.gupta@nii.ac.in

${ }^{\dagger}$ These authors have contributed equally to this work

‡These authors have contributed equally to this work

Specialty section: This article was submitted to Viral Immunology, a section of the journal

Frontiers in Immunology

Received: 02 December 2020 Accepted: 25 February 2021

Published: 11 March 2021

Citation: Ansari A, Arya R, Sachan S, Jha SN, Kalia A, Lall A, Sette A, Grifoni A, Weiskopf D, Coshic $P$, Sharma $A$ and Gupta N (2021) Immune Memory in Mild COVID-19 Patients and Unexposed Donors Reveals Persistent T Cell Responses After SARS-CoV-2 Infection. Front. Immunol. 12:636768. doi: 10.3389/fimmu.2021.636768

\section{Immune Memory in Mild COVID-19 Patients and Unexposed Donors Reveals Persistent T Cell Responses After SARS-CoV-2 Infection}

\author{
Asgar Ansari ${ }^{1+}$, Rakesh Arya ${ }^{2+}$, Shilpa Sachan ${ }^{1+}$, Someshwar Nath Jha ${ }^{1+}$, Anurag Kalia ${ }^{1+}$, \\ Anupam Lall ${ }^{3}$, Alessandro Sette ${ }^{4,5}$, Alba Grifoni $^{4}$, Daniela Weiskopf ${ }^{4}$, Poonam Coshic ${ }^{3 \neq}$, \\ Ashok Sharma $^{2 \neq}$ and Nimesh Gupta ${ }^{1 *}$

\begin{abstract}
${ }^{1}$ Vaccine Immunology Laboratory, National Institute of Immunology, New Delhi, India, ${ }^{2}$ Department of Biochemistry, All India Institute of Medical Sciences, New Delhi, India, ${ }^{3}$ Department of Transfusion Medicine, All India Institute of Medical Sciences, New Delhi, India, ${ }^{4}$ Center for Infectious Disease and Vaccine Research, La Jolla Institute for Immunology, La Jolla, CA, United States, ${ }^{5}$ Department of Medicine, Division of Infectious Diseases and Global Public Health, University of California, San Diego, La Jolla, CA, United States
\end{abstract}

Understanding the causes of the diverse outcome of COVID-19 pandemic in different geographical locations is important for the worldwide vaccine implementation and pandemic control responses. We analyzed 42 unexposed healthy donors and 28 mild COVID-19 subjects up to 5 months from the recovery for SARS-CoV-2 specific immunological memory. Using HLA class II predicted peptide megapools, we identified SARS-CoV-2 cross-reactive CD4 ${ }^{+} \mathrm{T}$ cells in around $66 \%$ of the unexposed individuals. Moreover, we found detectable immune memory in mild COVID-19 patients several months after recovery in the crucial arms of protective adaptive immunity; $\mathrm{CD} 4^{+} \mathrm{T}$ cells and $\mathrm{B}$ cells, with a minimal contribution from $\mathrm{CD}^{+} \mathrm{T}$ cells. Interestingly, the persistent immune memory in COVID-19 patients is predominantly targeted towards the Spike glycoprotein of the SARS-CoV-2. This study provides the evidence of both high magnitude pre-existing and persistent immune memory in Indian population. By providing the knowledge on cellular immune responses to SARS-CoV-2, our work has implication for the development and implementation of vaccines against COVID-19.

Keywords: human coronavirus, pre-existing immunity, CD4+ $\mathrm{T}$ cells, B cells, neutralizing antibody

\section{INTRODUCTION}

The COVID-19 pandemic has evolved with variable trajectory in diverse geographical locations. Pre-existing immunity acquired from 'common cold' Human Coronaviruses (HCoVs) could have substantial implication in the immunological and epidemiological outcome of the pandemic. Because of the diverse geo-distribution and prevalence of $\mathrm{HCoVs}$, there may be a varying impact of pre-existing immunity on the SARS-CoV-2 infection. Therefore, there is a considerable interest to understand the traits of pre-existing immunity and its impact on the virus spread and pathogenesis, disease outcome and the establishment of protective immunity in COVID-19. 
In context of pre-existing immunity, the cross-reactive $\mathrm{T}$ cells are the focus of extensive investigations. Recent reports reveal the existence of cross-reactive $\mathrm{CD}^{+}{ }^{+} \mathrm{T}$ cells in $\sim 20-50 \%$ of the individuals never been exposed to SARS-CoV-2 (1-5). These cross-reactive $\mathrm{CD} 4^{+} \mathrm{T}$ cells are largely canonical memory cells and they may be the outcome of previous infections with many of the common cold HCoVs (4). The cross-reactive memory CD4 T-cell subsets may lead to a favorable course of SARS-CoV-2 infection via direct anti-viral effects of CD4-CTL (Cytotoxic T Lymphocytes) or T helper cells, and also via establishing optimal germinal centers derived protective humoral immunity by follicular $\mathrm{T}$ helper cells. In fact, the cross-reactive immune memory to SARS-CoV-2 is limited to $\mathrm{CD}^{+} \mathrm{T}$ cells and more studies are required to understand the cross-reactivity from $\mathrm{HCoVs}$ in case of the humoral immunity (6-8). Most of these studies are limited to the antibody analyses and there is no firm knowledge available for the cross-reactivity in the B cell pool. The Spike glycoprotein of SARS-CoV-2 is the major target of neutralizing antibodies $(8,9)$. Particularly, antibodies targeting RBD display high neutralizing potential (10) and shown to be predicative of survival (11). However, there has been a concern over the decline of antibodies within first few months after SARS-CoV-2 infection $(12,13)$. Although, it's not clear if this decline is gradual and if the similar decline exists in the memory pool of T cells and B cells.

In addition to SARS-CoV-2, the cross-reactive immunity acquired from the common cold HCoVs may have substantial impact on the immune response to COVID-19 vaccine. Therefore, there is an urgent need to understand the attributes of pre-existing immunity and quality of protective immune memory in COVID-19 across the diverse populations. In this study, we have examined the traits and stability of immune memory in unexposed donors and patients recovered from mild COVID-19. We show that the SARS-CoV-2 cross-reactive antibodies and $\mathrm{CD}^{+} \mathrm{T}$ cells exist in the unexposed donors, with Non-spike domains as the predominant target of $\mathrm{CD} 4^{+} \mathrm{T}$ cells in $\sim 66 \%$ of the individuals. Moreover, we also show that immunological memory to SARS-CoV-2 is detectable in mild COVID-19 patients up to 5 months (median $\sim 3$ months) after recovery both in the $\mathrm{CD} 4^{+} \mathrm{T}$ cells and $\mathrm{B}$ cells. Interestingly, the durable immune memory in COVID-19 patients was highly targeted towards the Spike glycoprotein of the SARS-CoV-2. Our work provides the evidence of pre-existing reactivity and immune memory detectable in mild COVID-19 patients from the geographical location that is experiencing high burden of SARS-CoV-2 pandemic with an extremely low case fatality.

\section{MATERIALS AND METHODS}

\section{Ethics Statement}

This study was approved by the Institutional review boards of the National Institute of Immunology and All India Institute of Medical Sciences, New Delhi, India. Informed consent was obtained from all subjects during the enrolment. For analyses in healthy individuals, buffy coat and plasma samples isolated from blood of healthy donors were collected from the blood bank in All India Institute of Medical Sciences, New Delhi, India.

\section{PBMC Isolation}

For all samples blood was collected in K3 EDTA tubes (COVID19 donors) or EDTA coated blood bag (unexposed donors). Plasma was frozen at $-80^{\circ} \mathrm{C}$ in multiple aliquots. PBMCs were isolated using Ficoll Paque Plus (GE Life Sciences) density gradient medium and cryopreserved in multiple aliquots in Fetal Bovine Serum (Gibco) containing 10\% Dimethyl Sulfoxide (DMSO; Thermo-Fisher) and stored in liquid nitrogen until used in the assays. After revival, PBMCs were obtained with $>80 \%$ viability, as accessed by acridine orange and propidium iodide double staining using the LUNA-FL (Logos Biosystems Inc., USA) automated cell counter. Details of the study population are provided in Table $\mathbf{1}$.

\section{ELISA to Detect SARS-CoV-2 Specific IgG}

ELISA plates (Nunc, Maxisorp) were coated with 100 $\mathrm{l} /$ well of SARS-CoV-2 full length Spike protein (Native Antigen, UK) and Nucleoprotein (Sino Biologicals) in PBS ( $\mathrm{pH} \mathrm{7.4)} \mathrm{at} \mathrm{the} \mathrm{final}$ concentration of $1 \mu \mathrm{g} / \mathrm{ml}$ and incubated overnight at $4^{\circ} \mathrm{C}$. After wash, the plates were blocked with blocking buffer (PBS containing 3\% Skim milk and 0.05\% Tween-20) and incubated at room temperature (RT) for 2 hours. Plasma samples were heat inactivated at $56^{\circ} \mathrm{C}$ for 1 hour. Plates were washed and 3 -fold serially diluted heat inactivated plasma samples in dilution buffer (PBS containing 1\% Skim milk and 0.05\% Tween-20 in PBS) were added into the respective wells followed by incubation at RT for 1.5 hours. After incubation and wash, Goat anti-human IgG

TABLE 1 | Characteristics of COVID-19 Patients.

\begin{tabular}{lc}
\hline CovID-19 patients & $\mathbf{2 8}$ \\
\hline Age (Years) & $21-49($ median $=27)$ \\
Gender & \\
Male (\%) & $57.1 \%(16 / 28)$ \\
Female (\%) & $42.8 \%(12 / 28)$ \\
Residency & \\
New Delhi (India) & $100 \%(28 / 28)$ \\
SARS-CoV-2-PCR Positivity & $100 \%(28 / 28)$ \\
Disease Severity & \\
Mild & \\
Moderate & $78.6 \%(22 / 28)$ \\
Symptoms & $21.4 \%(6 / 28)$ \\
Fever & \\
Cough & $67.8 \%(19 / 28)$ \\
Sore throat & $60.7 \%(17 / 28)$ \\
Body ache & $75 \%(21 / 28)$ \\
Loss of taste & $67.8 \%(19 / 28)$ \\
Loss of smell & $75 \%(21 / 28)$ \\
Shortness in breath & $57.1 \%(16 / 28)$ \\
Respiratory distress & $21.4 \%(6 / 28)$ \\
Chest pain & $21.4 \%(6 / 28)$ \\
Blood in cough & $17.8 \%(5 / 28)$ \\
Vomiting/Nausea & $3.5 \%(1 / 28)$ \\
Diarrhea & $7.1 \%(2 / 28)$ \\
Days post diagnosis at collection & $10.7 \%(3 / 28)$ \\
Contact with known CoVID-19 patient & $82 \%(23 / 28)$ \\
&
\end{tabular}

${ }^{*}$ WHO Criteria. 
conjugated with Horseradish Peroxidase (HRP) (Southern Biotech) was added and plates were incubation at RT for 1 hour. The reaction was developed by adding ophenylenediamine dihydrochloride (OPD) peroxidase substrate (Sigma-Aldrich) for 10 minutes in dark at RT. The reaction was stopped by adding $50 \mu \mathrm{l} /$ well of $2 \mathrm{~N} \mathrm{HCl}$, followed by optical density (OD) measurement at $492 \mathrm{~nm}$ using MultiskanGO ELISA reader (Thermo-Fisher). The antigen coated wells that were added with sample diluent alone were used as blank to obtain the background OD values. For comparing the IgG titer in negative and COVID-19 recovered subjects, the Area Under Curve (AUC) was calculated for each specimen. The OD values obtained in test wells after subtracting the mean of background OD values were used for calculating the AUC, using a baseline of 0.05 for peak calculations. The positive response was defined as the value above the mean plus 3-times standard deviation of the lowest detected values, as in the case of reactivity with Spike protein, in all the tested samples from COVID-19 negative donors.

\section{ELISA to Detect HCoV-OC43 and HCoV-NL63 Specific IgG}

The IgG reactivity to the Nucleoprotein of $\mathrm{HCoV}-\mathrm{OC} 43$ and HCoV-NL63 was detected using in-house ELISA. The ELISA plates were coated with $100 \mu \mathrm{l} /$ well of $\mathrm{HCoVs}$ Nucleoprotein (Native Antigen, UK) in PBS at the final concentration of $1 \mu \mathrm{g} / \mathrm{ml}$ and incubated overnight at $4^{\circ} \mathrm{C}$. The plates were blocked and the reactivity was assessed in 1:100 diluted samples after incubation at RT for 1.5 hours. The Nucleoprotein-specific IgG were measured at $492 \mathrm{~nm}$ using the HRP-conjugated Goat antihuman IgG and OPD. The IgG reactivity was defined as the OD value in test wells after subtracting the mean of background OD values from blank wells.

\section{Virus Neutralization Assay}

The neutralization potential of the antibodies in unexposed and COVID-19 recovered subjects was assessed by using the SARSCoV-2 surrogate virus neutralization test (14). The test was performed following the manufacturer's instructions (Genscript). Briefly, plasma samples were incubated with the RBD-HRP and the mixture was captured on the plate coated with human ACE2. The reaction was developed using the TMB substrate and the absorbance was measured at $450 \mathrm{~nm}$ using a microplate reader. The sample absorbance was inversely proportional to the titre of the anti-SARS-CoV-2 neutralizing antibodies. The percent neutralization was calculated using the formula: (1-OD value of sample/OD value of Negative Control) $\mathrm{x}$ $100 \%$. The cut-off for the detection of SARS-CoV-2 neutralizing antibodies was determined by the manufacturer after validation with panel of confirmed COVID-19 patient sera and healthy control sera.

\section{Activation Induced Cell Marker (AIM) Assay for Quantification of CD4 ${ }^{+}$T Cells}

Antigen-specific CD4 T-cell analysis was performed using the sensitive AIM assay $(1,15,16)$. The PBMCs were stimulated for
24 hours in the presence of SARS-CoV-2 specific CD4 peptide megapools (MPs) (Spike protein: Spike; and remainder of the polyprotein: Non-spike) at $1 \mu \mathrm{g} / \mathrm{mL}$ in 96 -well $\mathrm{U}$ bottom plate in a total of $1 \times 10^{6}$ PBMCs per well. For negative control, an equimolar amount of DMSO (vehicle) was added to unstimulated well. Stimulation with cytomegalovirus CD4 MP (CMV, $1 \mu \mathrm{g} / \mathrm{mL}$ ), or Staphylococcal Enterotoxin B (SEB) were included as positive controls. After 24 hours, cells were washed with $1 \mathrm{~mL}$ of PBS with 2\% FBS (FACS buffer) and surface stained with antibody cocktail for 1 hour at $4^{\circ} \mathrm{C}$ in the dark; CD20, CD14, CD16, CD8a and fixable-viability dye coupled with APC eflour 780 in the dump channel, CD4-AlexaFluor 700 (RPA-T4), OX40-FITC (Ber-ACT35), CD137 PE Dazzle (4B4-1), CD45RA Brilliant Violet 785 (HI100) and CCR7 PE-Cy7 (3D12). Following the surface staining, cells were washed with FACS buffer and then fixed with freshly prepared $1 \%$ paraformaldehyde (Sigma Aldrich) for 30 minutes at $4^{\circ} \mathrm{C}$ in the dark. Cells were washed twice with FACS buffer and resuspended in FACS buffer before acquiring on a BD LSR Fortessa flow cytometer (BD Biosciences). Data were analysed using FlowJo 10.5.3. The positive response in the AIM assay was defined by setting up the limit of detection above the mean plus two-times of standard deviation of the response obtained in unstimulated conditions of all the unexposed and COVID-19 donors analysed. The frequency of responders to SARS-CoV-2 peptide pools was determined by applying the Fischer's exact test on the $\mathrm{AIM}^{+}$and $\mathrm{AIM}^{-}$cells in unstimulated and peptide stimulated conditions. Stimulation Index (SI) was calculated by dividing the percentage of $\mathrm{AIM}^{+}$cells after stimulation with peptide pools with the percentage of $\mathrm{AIM}^{+}$cells derived from DMSO stimulation. The SI $<1$ was depicted as 1 . The limit of positive stimulation index was defined as the median plus standard deviation of the lowest detected values, as in case of stimulation with Spike megapool, in COVID-19 unexposed donors.

\section{Activation Induced Cell Marker (AIM) Assay for Quantification of CD8 ${ }^{+}$T Cells}

The antigen-specific analyses of CD8 T cells were performed using the AIM assay, similar to the above mentioned CD4 ${ }^{+}$AIM assay. The PBMCs were stimulated with the class I peptide megapool consist of 628 peptides from the whole virus proteome and split into two megapool, CD8-A and CD8-B containing 314 peptide each, as detailed previously $(1,17)$. For negative control, an equimolar amount of DMSO (vehicle) was added to unstimulated well. Stimulation with Staphylococcal Enterotoxin B (SEB) were included as positive control. After stimulation, cells were washed and surface stained with antibody cocktail for 1 hour at $4^{\circ} \mathrm{C}$ in the dark; CD20, CD14, CD16, CD4 and fixable-viability dye coupled with APC eflour 780 in the dump channel, CD8-APC (RPA-T8), CD69-BV510 (FN50) and CD137 PE Dazzle (4B4-1). Following the surface staining the cells were washed, acquired and analysed as mentioned in the previous section of CD4-AIM assay. The positive response in the AIM assay was defined by setting up the limit of detection above the mean plus two-times of standard deviation of the response obtained in unstimulated controls of all 
the unexposed and COVID-19 donors analysed. Frequency of responders in unexposed and COVID-19 recovered subjects was determined using the combined data of CD8-A and CD8-B megapool after subtracting the background from the unstimulated controls.

\section{SARS-CoV-2 Specific B Cell ELISPOT}

The antigen-specific memory $B$ cells were measured in the cryopreserved PBMCs using polyclonal stimulation in RPMI 1640 in the presence of R848 $(1 \mu \mathrm{g} / \mathrm{mL})$ and IL-2 $(10 \mathrm{U} / \mathrm{mL})$ at cell density of $10^{6}$ PBMCs per well for 5 days. The Fluorospot plate (Mabtech) was charged with ethanol prior to the antigen coating. For antigen specific memory B cell analysis, plate was coated with SARS-CoV-2 full length Spike protein (Native Antigen, UK) and Nucleoprotein (Sino Biologicals) at concentration of $5 \mu \mathrm{g} / \mathrm{mL}$ and incubated overnight at $4^{\circ} \mathrm{C}$. As a control, for total memory B cell analysis, plates were coated with anti-human IgG, IgM, IgA coating antibodies (Mabtech) at concentration of $15 \mu \mathrm{g} / \mathrm{mL}$. Plates were washed and blocked with complete RPMI medium for at least 30 minutes at room temperature. Stimulated PBMCs were washed and seeded in complete RPMI at $0.5 \times 10^{6}-1 \times 10^{6}$ PBMCs per well for antigenspecific analysis and 20,000-50,000 cells for total B cell analysis. PBMCs were incubated at $37^{\circ} \mathrm{C}$ for 8 hours. Cells were discarded and plate was washed PBS. For detection of antibody secreting cell (ASC) spots, anti-human IgG-550, IgM-640 and IgA-490 detection antibodies (Mabtech) were added and plate was incubated for 2 hours at room temperature in dark. Plate was washed and fluorescence enhancer (Mabtech) was added to each well. ASC spots were detected on AID vSpot Spectrum Elispot/ Fluorospot reader system using AID Elispot software version 7.x. As no spots were detected in wells without the antigen, presence of a spot $>1$ in the antigen-coated well was considered as a positive response. ASC counts were normalized to ASCs per million of PBMCs for all analyses.

\section{Statistical Analysis}

In all experiments, data are expressed as the mean \pm s.e.m. The significance of the differences between the groups was analysed with the two-sided Mann-Whitney test, Fischer's exact test or Wilcoxon paired t-test as specified in the figure legends. $P$ values $<0.05$ were considered statistically significant. Statistical analyses were performed with the GraphPad Prism software version v8.

\section{RESULTS}

\section{Antibody Response in Unexposed Donors and Mild COVID-19 Recovered Patients With Synchronous Expansion of Antibodies to Hcov-OC43}

To investigate the quality and stability of immune memory in the COVID-19 patients we recruited 28 adult patients who had recovered from mild COVID-19 (Table 1). To explore the impact of cross-reactive immunity from 'common cold' coronaviruses we also utilized plasma samples and the peripheral blood mononuclear cells from 42 healthy blood donors collected prior to the pandemic during 2018-2019. The SARS-CoV-2 infection was diagnosed in all the recruited patients by viral PCR test. None of the patients required hospitalization and were quarantined with the mild-to-moderate manifestation of the disease. All the patients showed high titer IgG response to the full-length spike unlike unexposed donors that showed no evidence of spike-reactive IgG (Figures 1A, C). SARS-CoV-2 nucleoprotein-reactive IgG was present in 15 of the 42 unexposed donors tested, and the titer was significantly higher in the mild COVID-19 recovered patients (Figures 1B, D). We further analyzed neutralizing antibodies in 8 unexposed donors and 12 COVID-19 patients after $\geq 4$ months of recovery. We observed the presence of highly effective neutralizing antibodies in all the patients after the long duration of recovery (Figure 1E). Because we observed cross-reactive antibodies to the SARS$\mathrm{CoV}-2$ nucleoprotein in unexposed donors, we examined the IgG reactivity to nucleoproteins from common-cold $\mathrm{HCoV}$ OC43 and HCoV-NL63 as a representative betacoronavirus and alphacoronavirus, respectively. The IgG reactivity was present against both the $\mathrm{HCoV}-\mathrm{OC} 43$ and $\mathrm{HCoV}-\mathrm{NL} 63$ in almost all the unexposed donors. Interestingly, an increase in IgG reactivity in COVID-19 recovered patients was noted, but limited to the HCoV-OC43 (Figure 1F). Thus, the data suggest a detectable spike- and nucleoprotein-specific antibody response in the Indian patients recovered from mild disease, at least up to 5 months (median $\sim 3$ months) post COVID-19 diagnosis. Surprisingly, COVID-19 patients showed an increase in IgG response against the $\mathrm{HCoV}-\mathrm{OC} 43$ but not to the other common cold coronavirus tested, HCoV-NL-63, which may have less closely related Nucleoprotein to SARS-CoV-2 (18).

\section{Robust SARS-CoV-2 Specific CD4 ${ }^{+}$T-Cell Responses In Unexposed Donors and Mild COVID-19 Cases}

$\mathrm{CD} 4^{+} \mathrm{T}$ cells are crucial for both the optimal quality of antibodies and anti-viral responses. Thus, we examined the $\mathrm{CD}^{+} \mathrm{T}$ cell reactivity in unexposed donors and the patients recovered from mild COVID-19. We measured the SARS-CoV-2 specific CD $4^{+}$ $\mathrm{T}$ cells in the $\mathrm{T}$ cell receptor (TCR) dependent activation induced marker assay $(15,16)$. Here, we stimulated the PBMCs from 28 COVID-19 subjects and 32 unexposed healthy donors with the peptide megapool spanning the Spike domain (Spike) and the megapool covering the remainder of the SARS-CoV-2 genome (Non-spike) (1, 4). A CMV megapool and the Staphylococcus Enterotoxin B (SEB) superantigen was used as the positive control, while DMSO was used as the negative control (Figure $\mathbf{2 A}$ and Supplementary Figure 1).

A total of 7 out of 32 unexposed donors were associated with marginal frequency of SARS-CoV-2 spike-reactive $\mathrm{AIM}^{+}$ $\left(\mathrm{OX} 40^{+} \mathrm{CD} 137^{+}\right) \mathrm{CD}^{+} \mathrm{T}$ cells with an insignificant increase over the DMSO control (Figures 2B, D). Interestingly, 21 out of 32 unexposed donors robustly responded to the peptide megapool covering the Non-spike domains of virus with a significantly higher frequency of $\mathrm{AIM}^{+} \mathrm{CD}^{+} \mathrm{T}$ cells over the DMSO control (Figure 2B; DMSO vs Non-spike pool, $\mathrm{P}=0.0002$ 
A

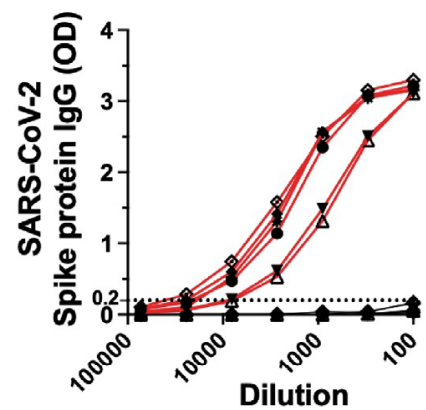

C

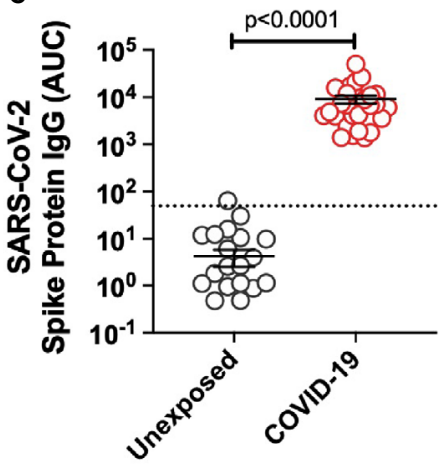

E

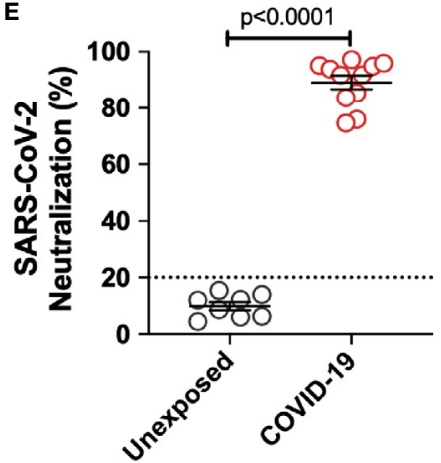

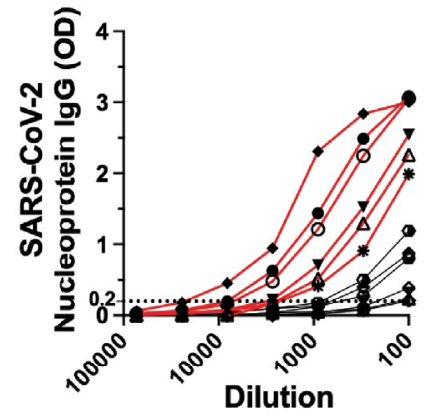

D

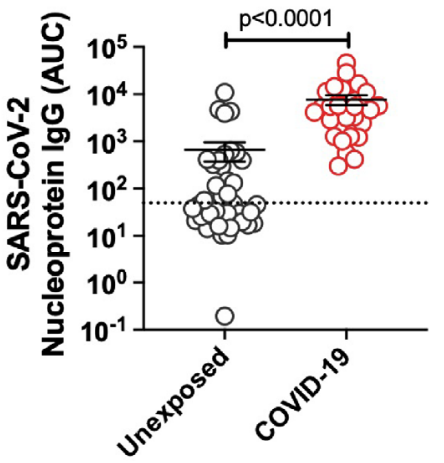

$\mathbf{F}$

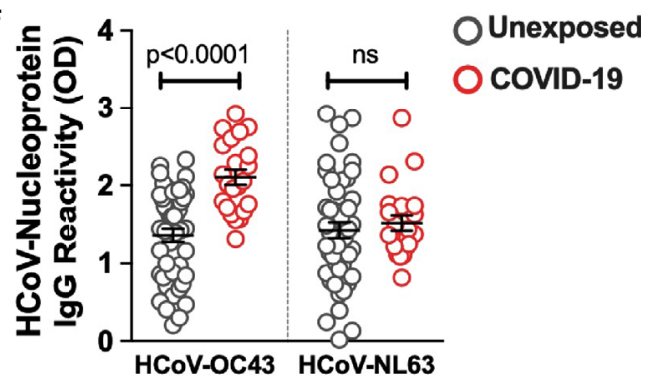

FIGURE 1 | SARS-CoV-2 IgG response in pre-pandemic unexposed donors and individuals recovered from mild COVID-19. The IgG titre was measured in plasma sample from unexposed donors collected prior to pandemic and the COVID-19 patients up to 5 months of recovery by ELISA using the full length Spike protein and Nucleoprotein. The ELISA curves in serially diluted samples are shown from 6 representative unexposed donors (grey line) and COVID-19 cases (red line) for (A) Spike protein, (B) Nucleoprotein. Area under the curve (AUC) for ELISA quantitation of the IgG binding to (C) Spike protein, (D) Nucleoprotein for 42 unexposed donors and 28 COVID-19 cases inclusive of 6 representative donors of each group shown in panel A and B. (E) Neutralizing antibody quantitation in unexposed donors $(n=8)$ and COVID-19 patients $(n=12)$ after $>4$ months recovery measured using the SARS-CoV-2 surrogate virus neutralization test. (F) HCoVs Nucleoprotein antigen binding (expressed as OD) assessed by ELISA in unexposed donors $(n=42)$ and COVID-19 recovered patients $(n=28)$. Black bars indicate the geometric mean. Dotted line in panels A-E represent the cut-off of positivity. Statistical comparisons were performed by two-tail Mann-Whitney test. ns: non-significant.

and Figure 2D). The unexposed donors consistently responded to the CMV peptide megapool and the SEB superantigen significantly over the DMSO control (Figure 2B; DMSO vs CMV pool, $\mathrm{P}=0.0005$; $\mathrm{DMSO}$ vs $\mathrm{SEB}, \mathrm{P}<0.0001$ ). The COVID19 recovered patients showed robust activation and detectable SARS-CoV-2-specific CD ${ }^{+} \mathrm{T}$ cells in response to the Spike (26/ 28; 93\%) (Figure 2C; DMSO vs Spike megapool, $\mathrm{P}<0.0001$ and Figure 2D) and to the Non-spike peptide pool $(24 / 28 ; 86 \%)$ (Figure 2C; DMSO vs Non-spike megapool, $\mathrm{P}<0.0001$ and
Figure 2D). Like unexposed donors, COVID-19 patients readily responded to the $\mathrm{CMV}$ peptide pool and $\mathrm{SEB}$ stimulation (Figure 2C; DMSO vs CMV pool, $\mathrm{P}=0.0003$; DMSO vs $\mathrm{SEB}, \mathrm{P}<0.0001)$. Moreover, no significant correlation was observed between the frequency of Spike-specific $\mathrm{CD} 4^{+} \mathrm{T}$ cells and the days from symptoms onset in convalescent patients (Supplementary Figure 2A). Next, we measured the stimulation index of antigen specific stimulations over the unstimulated DMSO control to quantify $\mathrm{CD} 4^{+} \mathrm{T}$ cell reactivity in case of 

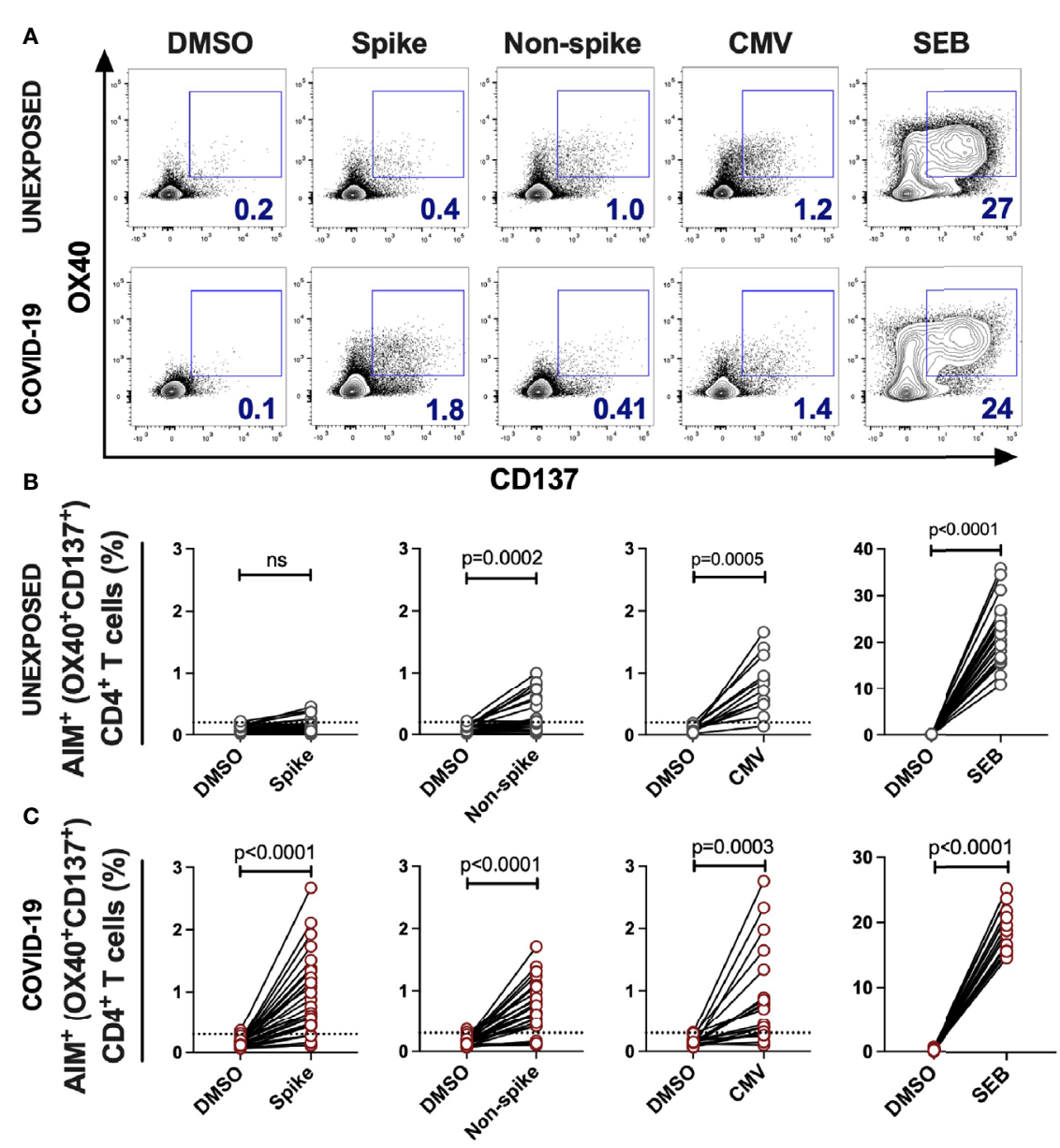

D
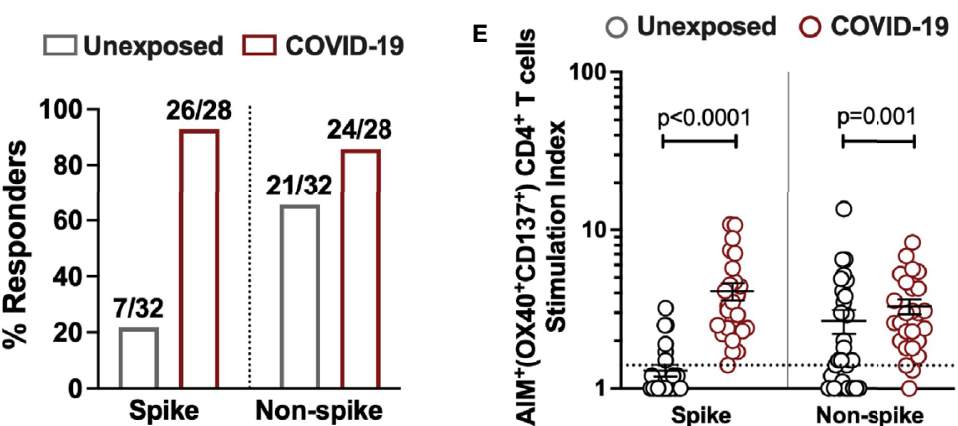

FIGURE 2 | SARS-CoV-2-specific CD4 ${ }^{+} T$ cells response in unexposed donors and recovered COVID-19 patients. The magnitude of SARS-CoV-2 specific CD4 ${ }^{+} T$ cells was determined in PBMCs collected from unexposed donors ("Unexposed", n=32) prior to pandemic and in COVID-19 patients ("COVID-19", n=28) up to 5 months of recovery. The PBMCs were stimulated with the peptide megapool specific to Spike glycoprotein (Spike) or to the remainder of the SARS-CoV-2 polyprotein (Non-spike). DMSO was used as the negative control, and CMV peptide megapool and SEB were used for positive stimulation controls. (A) Representative FACS contour plots of unexposed and COVID-19 patient in stimulation conditions of DMSO, Spike peptide megapool, Non-spike peptide megapool, $\mathrm{CMV}$ and SEB. Paired graphs depicting the reactivity of $\mathrm{AlM}^{+}\left(\mathrm{OX} 4 \mathrm{O}^{+} \mathrm{CD} 137^{+}\right) \mathrm{CD} 4^{+} \mathrm{T}$ cells between the negative control (DMSO) and antigen-specific stimulation in (B) Unexposed donors (C) COVID-19 patients. (D) Frequency of responders to Spike and Non-spike peptide pools in unexposed and COVID-19 recovered subjects as determined by the Fischer's exact test. The value on bars denote the number of responders/total number of donors tested. (E) Stimulation index quantitation of the $\mathrm{AlM}^{+}\left(\mathrm{OX} 4 \mathrm{O}^{+} \mathrm{CD} 137^{+}\right) \mathrm{CD}^{+} \mathrm{T}$ cells in Unexposed versus COVID-19 cases analysed in the same samples as in panel B and (C) Black bars indicate the geometric mean. Dotted line in panels B, C and E represent the limit of detection. Statistical comparisons were performed by (B, C) Wilcoxon paired t-test and (E) two-tail Mann-Whitney test. ns: non-significant. 
pre-existing immunity and in long-term post recovery from COVID-19. We observed a remarkably higher frequency of Spike-specific memory $\mathrm{CD} 4^{+} \mathrm{T}$ cells in recovered patients than the unexposed donors (Figure 2E; Unexposed vs COVID-19, $\mathrm{P}<0.0001)$. Surprisingly, higher magnitude of Non-spike reactive $\mathrm{CD}^{+} \mathrm{T}$ cells were also present in the unexposed donors as in recovered COVID-19 patients (Figure 2E; Unexposed vs COVID-19, $\mathrm{P}=0.001)$. Next, we determined the memory phenotype of the $\mathrm{CD}^{+} \mathrm{T}$ cells responding to the spike and non-spike peptide megapools (Supplementary Figure 3A). Both the central memory and effector memory compartments were mainly populated in antigen-specific $\mathrm{CD}^{+} \mathrm{T}$ cells, with no significant difference in the proportion specific to spike or non-spike genome of SARS-CoV-2 (Supplementary Figure 3B).

We further utilized the Class I peptide megapool to measure the SARS-CoV-2 specific $\mathrm{CD}^{+} \mathrm{T}$ cells in unexposed and recovered COVID-19 patients (Supplementary Figure 4A). The megapool consist of 628 peptides spanning the whole virus proteome and split into two pools, CD8-A and CD8-B, containing 314 peptides each (1). The minimal $\mathrm{CD}^{+} \mathrm{T}$ cell responses were detected only in the stimulation with CD8-A megapool, which consist of spike epitopes including the epitopes of other proteins (Supplementary Figures 4B, C). The unexposed donors and COVID-19 patients consistently responded to the SEB superantigen significantly over the DMSO control $(\mathrm{P}<0.0001$; Supplementary Figures $4 \mathrm{~B}, \mathrm{C})$. By combining the responses in both the megapool CD8-A and $\mathrm{CD} 8-\mathrm{B}$, total $\mathrm{CD}^{+} \mathrm{T}$ cell responses were detected in 2 of 18 unexposed donors and 4 of 18 recovered COVID-19 patients (Supplementary Figures 4 D, E).

Altogether, the antigen-specific $\mathrm{T}$ cell analyses suggest predominant and widespread $\mathrm{CD} 4^{+} \mathrm{T}$ cells responses over the $\mathrm{CD}^{+} \mathrm{T}$ cells in both the unexposed and recovered mild COVID19 patients. There was a minimal presence of Spike-specific $\mathrm{CD}^{+} \mathrm{T}$ cells in unexposed donors with a remarkably high magnitude in case of recovery from mild COVID-19. Interestingly, almost similar magnitude of non-spike specific $\mathrm{CD}^{+} \mathrm{T}$ cells are present in majority of the unexposed and COVID-19 recovery patients. Detection of Spike-specific memory $\mathrm{CD}^{+} \mathrm{T}$ cells several months after infection is encouraging for the efforts focusing on SARS-CoV-2 Spike protein as a vaccine candidate.

\section{High Magnitude Spike-Specific B Cells in Mild COVID-19 Recovered Subjects}

Because the mild COVID-19 patients showed robust Spikespecific $\mathrm{CD}^{+} \mathrm{T}$ cells reactivity, we examined if a similar finding would extend to SARS-CoV-2 B cell responses. Thus, utilizing SARS-CoV-2 Spike protein and Nucleoprotein (representative of Non-spike domains), we analyzed the frequency of each isotype-specific antibody secreting B cell population in unexposed subjects and the COVID-19 patients up to 5 months of recovery from mild disease (Figure 3A). The magnitude of IgG antibody secreting cells (ASC) was the highest among three subsets analyzed, as seen in the patients $\sim 4$ weeks after recovery (19). Surprisingly, all the patients showed significant 6-fold higher Spike-specific IgG-ASC over the ASCs specific to Nucleoprotein (Figure 3B; Count $/ 10^{6}$ PBMCs: Spike $780 \pm 84$, Nucleoprotein - 131 $\pm 35 ; \mathrm{P}<0.0001)$. The IgG-ASCs were also detected in around 6 (Spike-specific) and 14 (Nucleoprotein-specific) of the 28 unexposed subjects, with the substantially lower frequency than the COVID-19 patients (Figure 3B). Although the frequency of Nucleoprotein- and Spike-specific IgM-ASCs were significantly higher than the unexposed subjects, it was not significantly different in the COVID-19 recovered patients (Count/106 PBMCs: Spike 427 \pm 70 , Nucleoprotein - 463 \pm 76 ) (Figure 3C). Plasma cells secreting IgA were present in the least frequency in COVID-19 recovered patients and was only detected in the 8 (Spike-specific) and 10 (Nucleoprotein-specific) of the 28 unexposed subjects. Unlike Spike-specific IgA-ASCs that were detected in all the recovered patients, the Nucleoprotein specific IgA-ASCs were present in 13 of the 18 donors tested. However, like IgG-ASCs, Spike-specific memory IgA-ASCs were present in 2-fold higher frequency than the Nucleoprotein-specific cells in COVID-19 patients (Figure 3D; Count $/ 10^{6}$ PBMCs: Spike - 65 \pm 12 ,

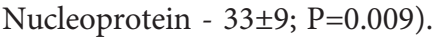

Altogether, these results indicate the existence of high magnitude IgG secreting cells in the antigen specific B-cell pool of mild COVID-19 patients. A small fraction of unexposed subjects showed cross-reactive ASCs present in a very low frequency. Like in the case of $\mathrm{CD} 4^{+} \mathrm{T}$ cells, a significant number of $\mathrm{B}$ cells is found in long-term after recovery from mild COVID-19, targeted towards the Spike protein of SARS-CoV-2.

\section{DISCUSSION}

Here, we report the extent of pre-existing immunity and immune memory in individuals from 2 to 5 months (median $\sim 3$ months) after the diagnosis of COVID-19. The existence of high titer Spike- and Nucleoprotein-specific IgG after several months postinfection indicates persistent antibody response in mild disease. Our observation is consistent with the recent reports where no decline was observed in antibodies to SARS-CoV-2 within 4 to 5 months of the COVID-19 diagnosis $(10,20)$. This is important for the vaccine development as the mild disease may provide the crucial knowledge for generating a long-term sustainable antibody response.

In our cohort, SARS-CoV-2 Spike cross-reactive antibodies were not detected in the unexposed donors' samples. This may be due to highly divergent Spike of SARS-CoV-2 than the seasonal coronaviruses (21). By contrast, almost $35 \%$ of the unexposed adult donors showed the existence of SARS-CoV-2 Nucleoprotein reactive antibodies. Unlike Spike protein, Nucleoprotein antibodies are more cross-reactive within the subgroups of HCoVs (22) and it's likely that the adult population in India has been exposed to common cold HCoVs as frequently as in the case with children and adolescents (23). The apparent nucleoprotein cross-reactivity seems to best correlate with the $\mathrm{HCoV}-\mathrm{OC} 43$ Nucleoprotein-specific antibodies as increased titers associated with SARS-CoV-2 


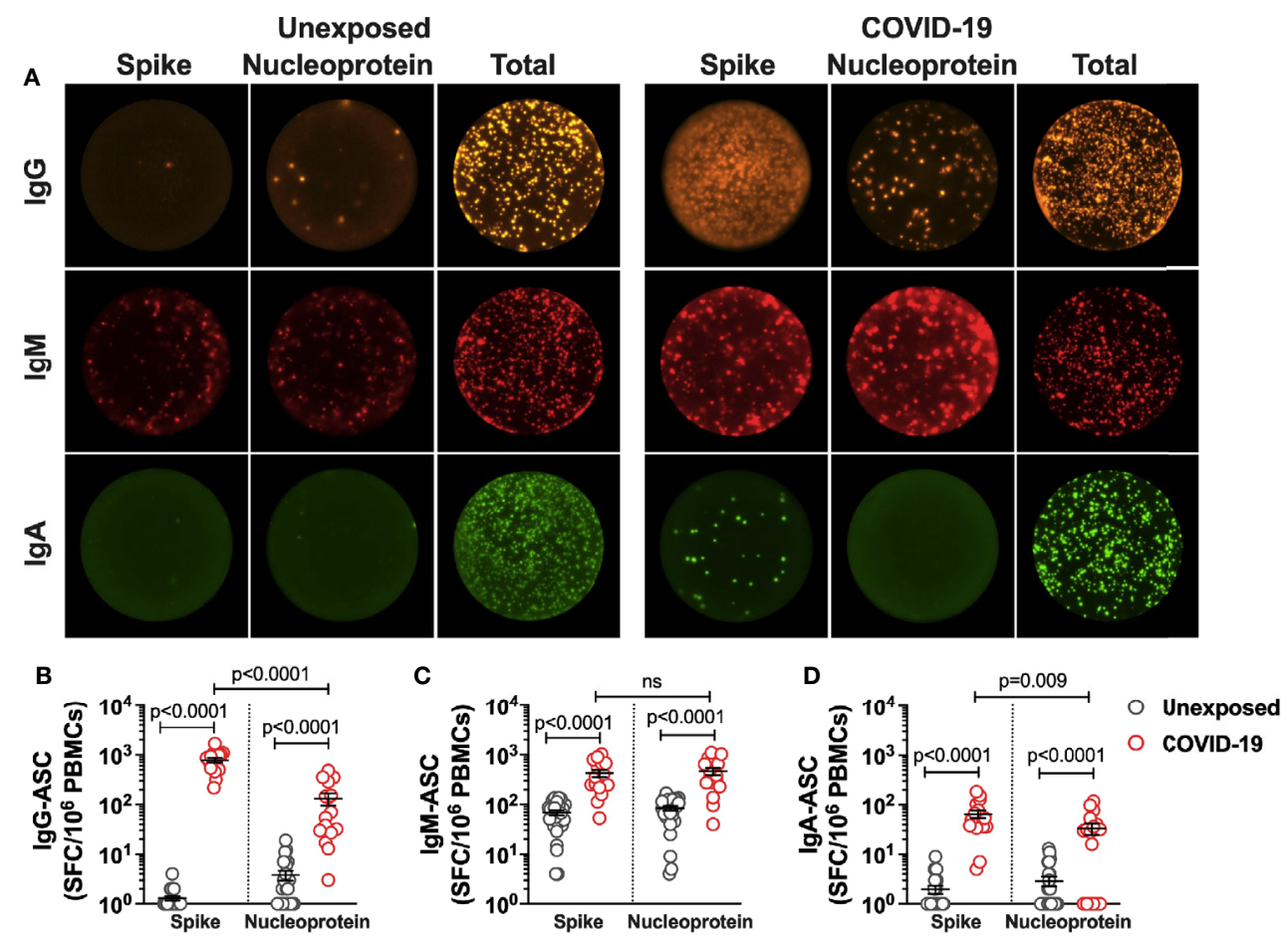

FIGURE 3 | SARS-CoV-2-specific memory B cells in recovered COVID-19 patients. The frequency and isotype distribution of antibody secreting B cells (ASC) was measured in the unexposed subjects ("Unexposed", n=28) prior to pandemic and in patients ("COVID-19", n=18) up to 5 months of recovery from mild COVID-19. The memory B cells in PBMCs were polyclonally stimulated before measuring the frequency of SARS-CoV-2 Spike glycoprotein- and Nucleoprotein-specific lgG, IgM and IgA antibody secreting cells in Fluorospot assay. (A) Representative images of IgG, IgM and IgA secreting B cells in Unexposed subject and recovered COVID19 patient. Graphs depicting the magnitude of antibody secreting B cells specific to the SARS-CoV-2 Spike glycoprotein and Nucleoprotein (expressed as spot forming cells (SFC) in $10^{6}$ PBMCs) for (B) IgG-ASC (C) IgM-ASC and (D) IgA-ASC, in Unexposed subjects (grey circle) and COVID-19 patients (red circle). For log scale, the spot count of less than one is depicted as 1. Black bars indicate the geometric mean. Statistical comparisons were performed by two-tail Mann-Whitney test. ns: non-significant.

infection were observed with $\mathrm{HCoV}-\mathrm{OC} 43$, a representative betacoronavirus used in this study. This may be due to more conserve Nucleoprotein immunodominant regions within the same family of betacoronaviruses (24). Although, it's not clear if the similar or the unique epitopes of $\mathrm{HCoV}$-OC43 are associated with this observed expansion. Indeed, the high titer Nucleoprotein targeting antibodies in unexposed donors and in long-term after recovery warrants detailed study to identify their implication in the SARS-CoV-2 pathogenesis and the disease outcome.

There is no information available on the pre-existing crossreactive $\mathrm{T}$ cells in the Indian population. We show that the crossreactivity to SARS-CoV-2 as well as the memory responses are mostly associated with the $\mathrm{CD} 4^{+} \mathrm{T}$ cells with a minor contribution from $\mathrm{CD}^{+} \mathrm{T}$ cells. The minimal contribution of $\mathrm{CD}^{+} \mathrm{T}$ cells among the cross-reactive $\mathrm{T}$ cells was also observed in other cohorts (1). The lack of SARS-CoV-2 specific $\mathrm{CD}^{+} \mathrm{T}$ cells in majority of the patients recovered from mild disease may be due to the poor stability or due to an inefficient establishment of the memory $\mathrm{CD}^{+} \mathrm{T}$ cells. Future investigations in different disease outcome across diverse populations are necessary to understand the implication of $\mathrm{CD}^{+} \mathrm{T}$ cells in SARS-CoV-2 pathogenesis. Our observation of poorly detected SARS-CoV-2
Spike-reactive $\mathrm{CD}^{+} \mathrm{T}$ cells in $\sim 20 \%$ of unexposed donors is consistent with the findings in the USA and the German cohorts $(1,3)$. However, higher frequency of SARS-CoV-2 Non-spike specific $\mathrm{CD}^{+} \mathrm{T}$ cells were observed in $\sim 66 \%$ of donors prior to the pandemic as compared to $\sim 50 \%$ in USA and the Singapore cohort $(1,2)$. In the Non-spike peptide megapool, the Nucleoprotein is the mainly targeted structural domain by the cross-reactive $\mathrm{CD} 4^{+} \mathrm{T}$ cells (4). Because of substantial homology of Nucleoprotein between common cold HCoVs and SARSCoV-2 and due to high prevalence of related common cold $\mathrm{HCoVs}$, as supported by the IgG reactivity, it's plausible that a higher extent of Nucleoprotein cross-reactive $\mathrm{CD} 4^{+} \mathrm{T}$ cells are present in our cohort of unexposed donors. Certainly, in-depth analyses in the unexposed donors are necessary to reveal if the prevalence and frequency of common cold HCoVs defines the targets of cross-reactivity to SARS-CoV-2 genome.

The cross-reactive $\mathrm{CD} 4^{+} \mathrm{T}$ cells might not be implicated solely in terminating the virus infection however they may limit the virus burden and reduce the course of symptomatic infection leading to lower incidences of severe disease (25). This is particularly interesting in context of the high frequency Nucleoprotein-specific cross-reactive $\mathrm{CD}^{+} \mathrm{T}$ cells. Nucleoprotein is the first and most abundantly produced 
multifunctional protein in the virus infected cells (26). The preexisting cross-reactive $\mathrm{CD} 4^{+} \mathrm{T}$ cells may limit the virus spread by cytolysis of the infected cells that are displaying the processed Nucleoprotein on their surface early in the infection. By controlling the virus spread and reducing the virus burden, pre-existing cross-reactive $\mathrm{CD}^{+}{ }^{+} \mathrm{T}$ cells might be implicated in providing a competitive window to the host to initiate an optimal protective immune response against the SARS-CoV-2.

Interestingly, high magnitude of Spike- and remainder of the genome $\mathrm{CD}^{+} \mathrm{T}$ cell responses are present in the patients long after recovery from mild COVID-19. However, unlike Spikespecific $\mathrm{CD}^{+}{ }^{+} \mathrm{T}$ cells that show a substantially higher magnitude over the cross-reactive $\mathrm{T}$ cells in unexposed donors, the non-spike specific memory $\mathrm{CD} 4^{+} \mathrm{T}$ cells are associated with a lesser increase after COVID-19. Future studies may reveal if there is an influence of cross-reactive memory $\mathrm{T}$ cells on de novo generation of noncross-reactive clones targeting the Non-spike domains $(27,28)$ or this diverse outcome is due to an immunodominance of $\mathrm{CD} 4^{+} \mathrm{T}$ cells targeting the highly immunodominant Spike glycoprotein leading to de novo expansion of Spike-specific $\mathrm{CD} 4^{+} \mathrm{T}$ cells and outcompeting the expansion of $\mathrm{CD}^{+} \mathrm{T}$ cells targeting low frequency $\mathrm{T}$-cell epitopes present in Nucleoprotein and the remainder of the genome $(4,17,29)$. Interestingly, in the similar lines, higher magnitude of Spike-specific IgG and IgA secreting B cells over Nucleoprotein-specific B cells further supports the notion of targeted and persistent immune response to a highly immunodominant Spike glycoprotein of SARS-CoV-2 in mild disease. The IgM secreting B cells were present at lower frequency than IgG secreting B cells in COVID19 recovery. However, these IgM secreting B cells were also detected in all the unexposed subjects. It's plausible that these IgM secreting B cells were not antigen-selected and developed in absence of a productive germinal center reaction during previous infection with the closely related human common cold coronaviruses (30). More studies in longitudinal prospective cohort are necessary to reveal the implication of pre-existing IgM secreting $\mathrm{B}$ cells in the SARS-CoV-2 pathogenesis and to determine if lower frequency of IgM secreting B cells is due to a poor stability in long-term or it's due to limited de novo generation in response to the SARS-CoV-2.

The limitations in our study include the sample size and the longitudinal sampling to probe the stability in immunological memory. As this study was of exploratory nature, the 28 longterm recovery samples represent the recruitment in a reasonable timeframe. In fact, the sample size was sufficient to determine the existence of cross-reactive $\mathrm{CD}^{+} \mathrm{T}$ cells and to reveal the persistence of memory $\mathrm{CD} 4^{+} \mathrm{T}$ cells in several months after recovery from COVID-19. Besides, the predicted epitopes utilized in this study to examine the $\mathrm{CD} 4^{+} \mathrm{T}$ cells may not cover the responses to all the epitopes in viral genome. However, these predicted peptide pools cover most of the immunodominant epitopes and provide an opportunity to detect the virus-specific $\mathrm{CD}^{+} \mathrm{T}$ cells in limitedly available patient blood sample. Certainly, further studies in long-term after recovery in a larger longitudinal cohort will be helpful in defining the breadth and durability of SARS-CoV-2 reactive memory $\mathrm{CD}^{+} \mathrm{T}$ cells.
Moreover, it will be very important to determine if similar characteristics of memory $\mathrm{CD}^{+}{ }^{+} \mathrm{T}$ cells exist in the recovery from different outcomes of disease from the asymptomatic to the severe COVID-19. While our work was in review, the knowledge on multiple virus variants emerged in the literature. Because the peptide pools used in our study originates from the reference strain of SARS-CoV-2 (GenBank: MN908947), the current analyses do not provide the information on reactivity of $\mathrm{T}$ cells to the mutated epitopes in recent virus variants. However, this remains an area of the future study to determine the capability of memory $\mathrm{T}$ cells established against the previously circulating virus in responding to the respective mutated epitopes in the recently emerged virus variants.

In summary, we show that the individuals recovered from mild disease display a response detectable several months after recovery in two crucial arms of protective immunity - $\mathrm{CD} 4^{+} \mathrm{T}$ cells and B cells. We also show the existence of pre-existing immunity in the unexposed donors, which is predominantly associated with the non-spike part of the genome of SARS$\mathrm{CoV}-2$. Although the cross-reactive $\mathrm{T}$ cells are present against both the spike and non-spike epitopes, the magnitude of crossreactive $\mathrm{CD}^{+} \mathrm{T}$ cells targeting the non-spike epitopes is extremely high in our cohort. Indian continent has seen high burden of the COVID-19 incidences; however, the case fatality rates are extremely low. Whether high magnitude of crossreactive $\mathrm{CD}^{+} \mathrm{T}$ cells are contributing to this less severe outcome needs to be addressed in the prospective cohort before and after COVID-19. The knowledge on implication of cross-reactive $\mathrm{CD}^{+} \mathrm{T}$ cells in the disease outcome and in establishment of immunological memory is crucial for the development and implementation of COVID-19 vaccines.

\section{DATA AVAILABILITY STATEMENT}

The original contributions presented in the study are included in the article/Supplementary Material. Further inquiries can be directed to the corresponding author.

\section{ETHICS STATEMENT}

The studies involving human participants were reviewed and approved by the Institutional review boards of the National Institute of Immunology and All India Institute of Medical Sciences, New Delhi, India. The patients/participants provided their written informed consent to participate in this study.

\section{AUTHOR CONTRIBUTIONS}

RA, JL, AsS, and PC: Enrolled and categorized subjects, collected samples and provided clinical information. AA, SS, SJ, and AK: performed experiments. AlS, AG, and DW: Contributed essential material. NG: Conceived and supervised the study, analysed the data and wrote the manuscript. AlS and DW: critically reviewed 
the manuscript. All authors contributed to the article and approved the submitted version.

\section{FUNDING}

This work was supported by Science and Engineering Research Board, DST grant IPA/2020/000077 (to NG, AsS, PC), Biotechnology Industry Research Assistance Council, DBT grant BT/COVID0010/01/20 (to NG). Further support provided from NIH contract 75N9301900065 (to AlS, DW) and NIH grant U01 (U01AI141995-03) to AlS.

\section{REFERENCES}

1. Grifoni A, Weiskopf D, Ramirez SI, Mateus J, Dan JM, Moderbacher CR, et al. Targets of T Cell Responses to SARS-CoV-2 Coronavirus in Humans with COVID-19 Disease and Unexposed Individuals. Cell (2020) 181(7):1489501.e15. doi: 10.1016/j.cell.2020.05.015

2. Le Bert N, Tan AT, Kunasegaran K, Tham CYL, Hafezi M, Chia A, et al. SARSCoV-2-specific T cell immunity in cases of COVID-19 and SARS, and uninfected controls. Nature (2020) 584(7821):457-62. doi: 10.1038/s41586-020-2550-Z

3. Braun J, Loyal L, Frentsch M, Wendisch D, Georg P, Kurth F, et al. SARSCoV-2-reactive $\mathrm{T}$ cells in healthy donors and patients with COVID-19. Nature (2020) 587(7833):270-74 . doi: 10.1038/s41586-020-2598-9

4. Mateus J, Grifoni A, Tarke A, Sidney J, Ramirez SI, Dan JM, et al. Selective and cross-reactive SARS-CoV-2 T cell epitopes in unexposed humans. Science (2020) 370(6512):89-94. doi: 10.1126/science.abd3871

5. Weiskopf D, Schmitz KS, Raadsen MP, Grifoni A, Okba NMA, Endeman H, et al. Phenotype and kinetics of SARS-CoV-2-specific T cells in COVID-19 patients with acute respiratory distress syndrome. Sci Immunol (2020) 5 (48):1-10. doi: 10.1126/sciimmunol.abd2071

6. Yuan M, Wu NC, Zhu X, Lee CD, So RTY, Lv H, et al. A highly conserved cryptic epitope in the receptor binding domains of SARS-CoV-2 and SARSCoV. Science (2020) 368(6491):630-3. doi: 10.1126/science.abb7269

7. Wec AZ, Wrapp D, Herbert AS, Maurer DP, Haslwanter D, Sakharkar M, et al. Broad neutralization of SARS-related viruses by human monoclonal antibodies. Science (2020) 369(6504):731-6. doi: 10.1126/science.abc7424

8. Premkumar L, Segovia-Chumbez B, Jadi R, Martinez DR, Raut R, Markmann $\mathrm{A}$, et al. The receptor binding domain of the viral spike protein is an immunodominant and highly specific target of antibodies in SARS-CoV-2 patients. Sci Immunol (2020) 5(48):1-9. doi: 10.1126/sciimmunol.abc8413

9. Walls AC, Park YJ, Tortorici MA, Wall A, McGuire AT, Veesler D. Structure, Function, and Antigenicity of the SARS-CoV-2 Spike Glycoprotein. Cell (2020) 181(2):281-92.e6. doi: 10.1016/j.cell.2020.02.058

10. Wajnberg A, Amanat F, Firpo A, Altman DR, Bailey MJ, Mansour M, et al. Robust neutralizing antibodies to SARS-CoV-2 infection persist for months. Science (2020) 370(6521):1227-30. doi: 10.1126/science.abd7728

11. Secchi M, Bazzigaluppi E, Brigatti C, Marzinotto I, Tresoldi C, Rovere-Querini $\mathrm{P}$, et al. COVID-19 survival associates with the immunoglobulin response to the SARS-CoV-2 spike receptor binding domain. J Clin Invest (2020) 130 (12):6366-78. doi: 10.1172/JCI142804

12. Seow J, Graham C, Merrick B, Acors S, Pickering S, Steel KJA, et al. Longitudinal observation and decline of neutralizing antibody responses in the three months following SARS-CoV-2 infection in humans. Nat Microbiol (2020) 5(12):1598-607. doi: 10.1038/s41564-020-00813-8

13. Long QX, Tang XJ, Shi QL, Li Q, Deng HJ, Yuan J, et al. Clinical and immunological assessment of asymptomatic SARS-CoV-2 infections. Nat Med (2020) 26(8):1200-4. doi: 10.1038/s41591-020-0965-6

14. Tan CW, Chia WN, Qin X, Liu P, Chen MI, Tiu C, et al. A SARS-CoV-2 surrogate virus neutralization test based on antibody-mediated blockage of ACE2-spike protein-protein interaction. Nat Biotechnol (2020) 38(9):1073-8. doi: $10.1038 /$ s41587-020-0631-z

\section{ACKNOWLEDGMENTS}

We are thankful to all the patients for generous support in this study, and Mr. Neeraj, Mr. Jagat Singh (AIIMS) and Mr. Sudipta Das (NII) for technical support.

\section{SUPPLEMENTARY MATERIAL}

The Supplementary Material for this article can be found online at: https://www.frontiersin.org/articles/10.3389/fimmu.2021. 636768/full\#supplementary-material

15. Havenar-Daughton C, Reiss SM, Carnathan DG, Wu JE, Kendric K, Torrents de la Pena A, et al. Cytokine-Independent Detection of Antigen-Specific Germinal Center T Follicular Helper Cells in Immunized Nonhuman Primates Using a Live Cell Activation-Induced Marker Technique. J Immunol (2016) 197(3):994-1002. doi: 10.4049/jimmunol.1600320

16. Reiss S, Baxter AE, Cirelli KM, Dan JM, Morou A, Daigneault A, et al. Comparative analysis of activation induced marker (AIM) assays for sensitive identification of antigen-specific CD4 T cells. PloS One (2017) 12(10): e0186998. doi: 10.1371/journal.pone.0186998

17. Grifoni A, Sidney J, Zhang Y, Scheuermann RH, Peters B, Sette A. A Sequence Homology and Bioinformatic Approach Can Predict Candidate Targets for Immune Responses to SARS-CoV-2. Cell Host Microbe (2020) 27(4):671-80 e2. doi: 10.1016/j.chom.2020.03.002

18. Huang AT, Garcia-Carreras B, Hitchings MDT, Yang B, Katzelnick LC, Rattigan SM, et al. A systematic review of antibody mediated immunity to coronaviruses: kinetics, correlates of protection, and association with severity. Nat Commun (2020) 11(1):4704. doi: 10.1038/s41467-020-18450-4

19. Juno JA, Tan HX, Lee WS, Reynaldi A, Kelly HG, Wragg K, et al. Humoral and circulating follicular helper $\mathrm{T}$ cell responses in recovered patients with COVID-19. Nat Med (2020) 26(9):1428-34. doi: 10.1038/s41591-020-0995-0

20. Gudbjartsson DF, Norddahl GL, Melsted P, Gunnarsdottir K, Holm H, Eythorsson E, et al. Humoral Immune Response to SARS-CoV-2 in Iceland. N Engl J Med (2020) 383(18):1724-34. doi: 10.1056/NEJMoa2026116

21. Forni D, Cagliani R, Clerici M, Sironi M. Molecular Evolution of Human Coronavirus Genomes. Trends Microbiol (2017) 25(1):35-48. doi: 10.1016/ j.tim.2016.09.001

22. Agnihothram S, Gopal R, Yount BLJr, Donaldson EF, Menachery VD, Graham RL, et al. Evaluation of serologic and antigenic relationships between middle eastern respiratory syndrome coronavirus and other coronaviruses to develop vaccine platforms for the rapid response to emerging coronaviruses. J Infect Dis (2014) 209(7):995-1006. doi: 10.1093/ infdis/jit609

23. Ng KW, Faulkner N, Cornish GH, Rosa A, Harvey R, Hussain S, et al. Preexisting and de novo humoral immunity to SARS-CoV-2 in humans. Science (2020) 370(6522):1339-43. doi: 10.1126/science.abe1107

24. Meyer B, Drosten C, Muller MA. Serological assays for emerging coronaviruses: challenges and pitfalls. Virus Res (2014) 194:175-83. doi: 10.1016/j.virusres.2014.03.018

25. Lipsitch M, Grad YH, Sette A, Crotty S. Cross-reactive memory T cells and herd immunity to SARS-CoV-2. Nat Rev Immunol (2020) 20(11):709-13. doi: 10.1038/s41577-020-00460-4

26. de Wit E, van Doremalen N, Falzarano D, Munster VJ. SARS and MERS: recent insights into emerging coronaviruses. Nat Rev Microbiol (2016) 14 (8):523-34. doi: 10.1038/nrmicro.2016.81

27. Brehm MA, Pinto AK, Daniels KA, Schneck JP, Welsh RM, Selin LK. T cell immunodominance and maintenance of memory regulated by unexpectedly cross-reactive pathogens. Nat Immunol (2002) 3(7):627-34. doi: 10.1038/ ni806

28. Johnson LR, Weizman OE, Rapp M, Way SS, Sun JC. Epitope-Specific Vaccination Limits Clonal Expansion of Heterologous Naive T Cells during 
Viral Challenge. Cell Rep (2016) 17(3):636-44. doi: 10.1016/ j.celrep.2016.09.019

29. Olson MR, Chua BY, Good-Jacobson KL, Doherty PC, Jackson DC, Turner SJ. Competition within the virus-specific CD4 T-cell pool limits the $\mathrm{T}$ follicular helper response after influenza infection. Immunol Cell Biol (2016) 94(8):72940. doi: $10.1038 /$ icb.2016.42

30. Bohannon C, Powers R, Satyabhama L, Cui A, Tipton C, Michaeli M, et al. Long-lived antigen-induced IgM plasma cells demonstrate somatic mutations and contribute to long-term protection. Nat Commun (2016) 7:11826. doi: $10.1038 /$ ncomms 12687

Conflict of Interest: AlS is listed as inventor on patent application no. 63/ 012,902, submitted by La Jolla Institute for Immunology, that covers the use of the megapools and peptides thereof for therapeutic and diagnostic purposes.
AlS is a consultant for Gritstone, Flow Pharma, Merck, Epitogenesis, Gilead and Avalia.

The remaining authors declare that the research was conducted in the absence of any commercial or financial relationships that could be construed as a potential conflict of interest.

Copyright (c) 2021 Ansari, Arya, Sachan, Jha, Kalia, Lall, Sette, Grifoni, Weiskopf, Coshic, Sharma and Gupta. This is an open-access article distributed under the terms of the Creative Commons Attribution License (CC BY). The use, distribution or reproduction in other forums is permitted, provided the original author(s) and the copyright owner(s) are credited and that the original publication in this journal is cited, in accordance with accepted academic practice. No use, distribution or reproduction is permitted which does not comply with these terms. 